\title{
APLICAÇÃO DE CADEIAS DE MARKOV PARA ANALISAR A VARIAÇÃO DO PREÇO DO AÇO NO BRASIL
}

\begin{abstract}
Bruna Gabriele de Matos (UNIVERSIDADE DE CAXIAS DO SUL) bgmatos@ucs.br Rafaela Boeira Cechin (UNIVERSIDADE DE CAXIAS DO SUL) rbcechin@ucs.br Nathalia Tessari Moraes (UNIVERSIDADE DE CAXIAS DO SUL) ntmoraes@ucs.br Bruna Caroline Orlandin (UNIVERSIDADE DE CAXIAS DO SUL) bcorlandin@ucs.br Leandro Luis Corso (UNIVERSIDADE DE CAXIAS DO SUL) llcorso@ucs.br
\end{abstract}

\begin{abstract}
Resumo
Considerando que o aço é um dos principais ativos do Brasil, cabe salientar que a variação impacta em diversos setores. Atualmente, a indústria é fortemente afetada pelo valor deste produto, onde é bastante utilizado como matéria-prima. Para realizar uma avaliação da variação do preço se implementou um modelo de Cadeias de Markov em um banco de dados do valor médio do quilograma no Brasil. Por meio da técnica, e com base na avaliação do estado estável da base de dados evidenciou-se que as maiores probabilidades de ocorrência são de uma variação percentual lenta do valor, fato este que pode ser utilizado no planejamento de empresas. O estado estável do modelo mostra que a probabilidade de uma variação ser baixa é de aproximadamente $60 \%$ entre meses subsequentes. Desta forma, com base no estado atual se pode verificar as probabilidades de transição para os próximos meses.
\end{abstract}

Palavras-Chaves: Cadeias de Markov; Aço; Processos Estocásticos; Estado Estável.

\section{Introdução}

O preço das comodities no Brasil é formado, em grande parte das vezes, por um conjunto de fatores, mas dependem fortemente de bolsas do país, do exterior e também do dólar. A variação destes valores pode afetar diversos setores, como, por exemplo, o metalmecânico, que é fortemente impactado pelo valor do aço, minério de ferro entre outros. Sabe-se que o encarecimento do preço do aço pode representar a possibilidade de ocorrência de crises no setor metalmecânico e, ao mesmo tempo, pode encarecer produtos uma vez que eleva o custo produtivo. Devido a isso, no presente trabalho se buscou analisar o comportamento do preço médio do aço no Brasil. Desta forma, um banco de dados com preços de janeiro de 2010 a outubro de 2019 foi analisado.

Dentro da área de Pesquisa Operacional (PO), existe uma área de análise de processos estocásticos, onde dados com variação ao longo do tempo são estudados e analisados para aplicações práticas, grande parte das vezes. Dentre as técnicas está Cadeias de Markov, que teve sua origem onde o objetivo é descrever o comportamento de estados e a transição entre 
eles, podendo considerar diferentes distribuições de probabilidade (CRUZ, 2012; BOLSON et al., 2019). Somado a isso, sabe-se que aplicação de Cadeias de Markov em dados financeiros podem trazer diferentes tipos de benefícios para tomada de decisão (CECHIN; CORSO, 2019).

O objetivo deste trabalho é o cálculo da probabilidade de variação do preço médio do aço no Brasil, juntamente com o estado estável e o tempo esperado de recorrência, utilizando uma base histórica.

\section{Pesquisa operacional}

A histórica de PO é marcada pelos registros ao longo da Segunda Guerra Mundial (COLIN, 2007; TAHA, 2008), entretanto, os modelos de otimização já sejam utilizados há muito mais tempo. Os registros relatam decisões tomadas para modelar fenômenos matemáticos onde a estatística era, muitas vezes, fator fundamental a ser considerado. De fato, muitos problemas sem solução passaram a apresentar dados quantitativos, possibilitando a tomada de decisão (ANDREADE, 2015). Devido aos avanços apresentados, tomadas de decisões começaram a ser todas mesmo em problemas onde havia incerteza de informações, o que permitiu o desenvolvimento de robustos modelos matemáticos de otimização aplicados na atualidade (CORSO; WALLACE, 2015).

\subsection{Cadeias de Markov}

Atualmente, o método de Cadeias de Markov possui diferentes aplicações, como, por exemplo: variação do preço de comodities e também aplicações financeiras (CECHIN; CORSO, 2019). Hillier e Lieberman (2013) citam que Cadeias de Markov podem estudar casos particulares de processos estocásticos, onde o estado atual é suficiente para previsão do estado futuro. Para realizar qualquer tipo de previsão, é fundamental que os dados históricos sejam confiáveis (RAGSDALE, 2009). Como definição, um processo estocástico pode ser representando como um conjunto $\left\{X_{t}, t=0,1,2, \ldots\right\}$ com espaço de estado $\mathrm{E}=\{1,2, . ., \mathrm{s}\}$. Onde, a propriedade de Markov faz com que a soma dos termos de probabilidade da Equação 1 seja igual a 1, ao mesmo tempo, assumindo que todo $n$ é um número natural e todo $i$ pertencente ao conjunto $\mathrm{E}=\{1,2, \ldots \mathrm{s}\}$.

$P\left(X_{n}=i_{n} \mid X_{n-1}=i_{n-1}, \ldots, X_{0}=i_{0}\right)=P\left(X_{n}=i_{n} \mid X_{n-l}=i_{n-1}\right)$ 
Outra forma de representar a Cadeia de Markov é por meio do diagrama de transição, como apresentado na Figura 1, onde se apresentam 3 estados. Nesta mesma representação, tem-se $\mathrm{E}_{i}$ como os $i$ estados, mostrados em círculos e conectados por arestas. As probabilidades de transições, são mostradas por $\operatorname{arcos}\left(\mathrm{p}_{i j}\right)$, onde se presenta a probabilidade de sair de um estado $i$ e ir para um estado $j$ em um passo de tempo.

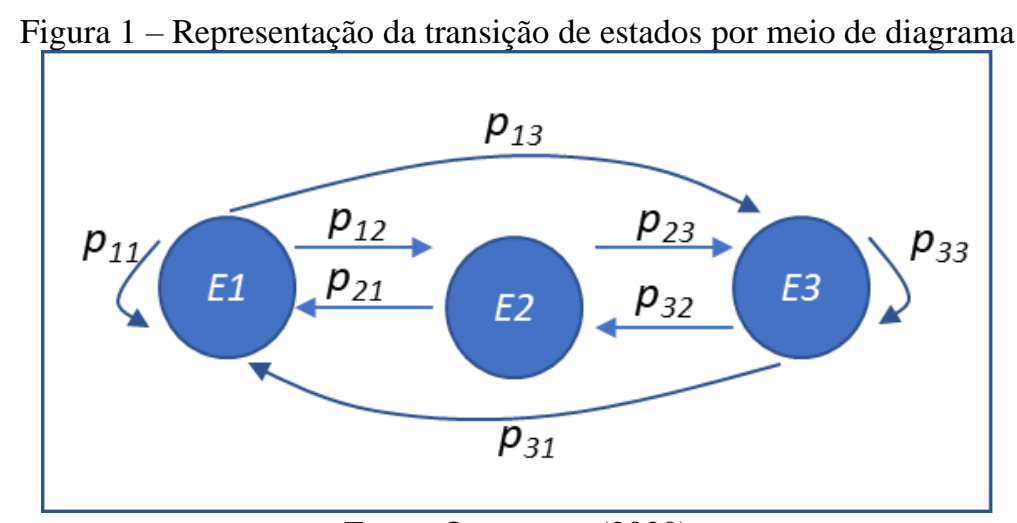

Fonte: Os autores (2020)

Dentre a utilização da técnica de Cadeias de Markov se pode mencionar a matriz de transição, que é a representação da transição entre estados. A forma padrão da matriz de $N$ estados pode ser dada por:

$P=\left[\begin{array}{cccc}p_{11} & p_{12} & \ldots & p_{1 N} \\ p_{21} & p_{22} & \ldots & p_{2 N} \\ \ldots & \ldots . & \ldots & \ldots \\ p_{N 1} & p_{N 2} & \ldots & p_{N N}\end{array}\right]$

Da mesma forma que o diagrama, tem-se a probabilidade de transição de ir de um estado $i$ para o estado $j$ dado por $p_{i j}$. É fundamental destacar que a soma dos termos da mesma deve ser igual a 1. Como apresenta Taha (2008):

$p_{i 1}+p_{i 2}+\ldots .+p_{i j}=1$

Outra propriedade considerada, é que a Cadeia de Markov é ergódica quando é possível de ir de um estado para outro em um determinado número de passos. Também, considera-se possível determinar o estado estável $\left(\pi_{j}\right)$ dos $j$ estados da Cadeia. As Equação 4 e 5 em conjunto representam um sistema de equações lineares que fazem parte da formulação do estado estável.

$\pi_{j}=\sum \pi_{i} p_{i j}$ 
Por meio desta propriedade é também possível calcular os períodos de tempo médios e necessários para sair de um estado e voltar para o estado inicial de partida. Este é chamado de tempo de recorrência esperado, e é dado por $\mu_{i i}$. Este termo está apresentado na Equação 6.

$$
\mu_{i i}=\frac{1}{\pi_{i}} \quad, \text { para } i=\{1,2, \ldots, N\}
$$

\section{Metodologia}

O desenvolvimento da metodologia contempla as principais etapas para utilização prática de Cadeias de Markov e estão apresentadas na Figura 2. Em seguida, cada uma das etapas é apresentada.

Figura 2: Etapas de desenvolvimento da metodologia

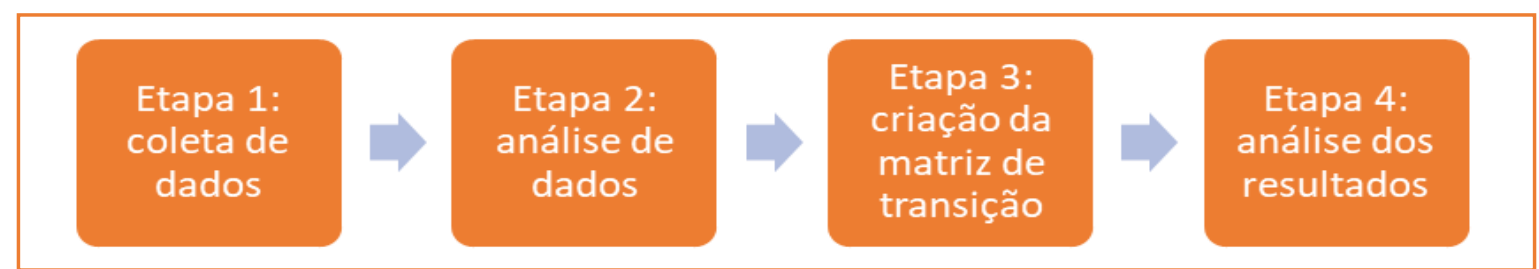

Fonte: Os autores (2020)

\subsection{Etapa 1 - Coleta de dados}

Os dados referentes ao preço do aço foram considerados de janeiro de 2010 a outubro de 2019, onde os valores trabalho são a média de todos os estados. A base de dados contemplou 118 meses na avaliação do preço médio do aço no Brasil. É importante ressaltar que cada estado pode apresentar os seus próprios valores para o preço do aço, o valor considerado nesta análise faz uso da média do valor de todos os estados.

\subsection{Etapa 2 - Análise dos dados}

Com a seleção dos dados dos preços realizadas, se passou para a avaliação da variação percentual dos preços médios mensais no Brasil. Os valores percentuais foram considerados para criação da matriz de transição. Depois disso, se partiu para o desenvolvimento da matriz de transição, apresentada na etapa seguinte. 


\subsection{Etapa 3 - Criação da matriz de transição}

Para a construção da matriz de transição se faz necessária a elaboração dos intervalos de variação. Desta forma, os dados foram avaliados, onde se buscaram o maior e o menor valor, a partir disso se fez a divisão em 5 faixas. Desta forma, buscou-se proporcionar uma análise visando probabilidades para aumento e decréscimo do valor do preço.

\subsection{Etapa 4 - Avaliação do estado estável}

A partir da criação da Matriz de transição é possível, por meio do sistema de equações lineares, apresentadas nas Equações 5 e 6 se obter os valores do estado estável. Para isso, o sistema de equações é resolvido e os valores das variáveis apresentados.

\section{Resultados obtidos}

Para o desenvolvimento do trabalho as etapas apresentadas na seção anterior foram executadas. Para a etapa 1, referente ao levantamento de dados, apresenta-se a Figura 3, onde tem-se o valor do preço do aço ao longo do período do tempo analisado. Para a etapa 2, referente a avaliação dos dados, calculou-se variação percentual mensal, e está apresentada na Figura 4.

Figura 3: Valor do preço do aço ao longo dos meses

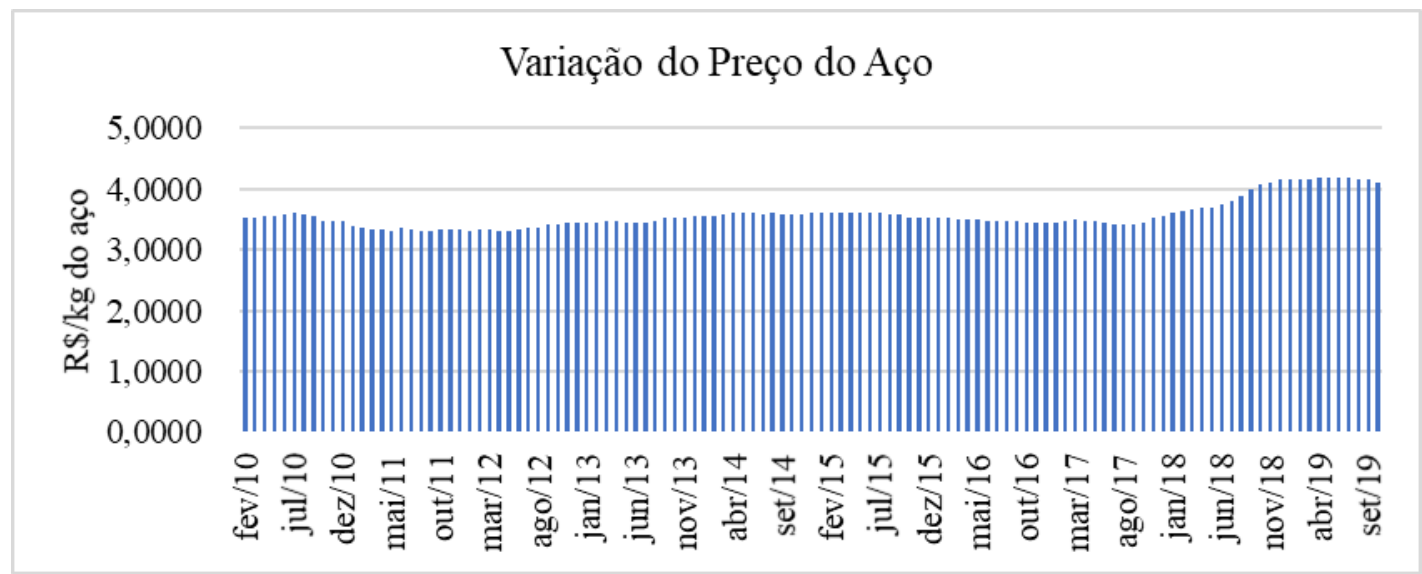

Fonte: Os autores (2020) 
Figura 4: Percentual de variação mensal do preço do aço ao longo dos meses

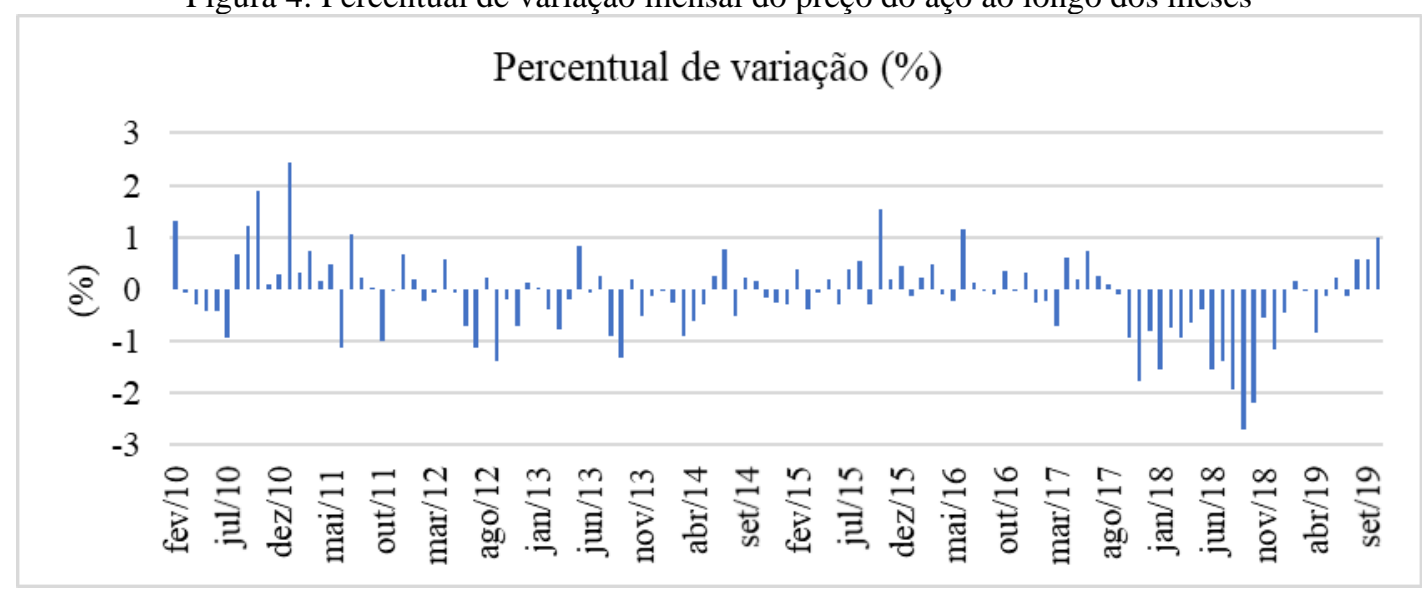

Fonte: Os autores (2020)

A partir disso, etapa 3, inicialmente se realizou a divisão das faixas. Esta divisão está apresentada na Tabela 1. A matriz de transição leva em conta a distribuição de dados apresentados na Equação 2.

Tabela 1 - Divisão da variação percentual para cada faixa

\begin{tabular}{ccccc}
\hline A & B & C & D & E \\
\hline$<-1,67 \%$ & $-1,67 \%$ a $-0,64 \%$ & $-0,64 \%$ a $0,38 \%$ & $0,39 \%$ a $1,41 \%$ & $>1,41 \%$ \\
\hline \multicolumn{6}{c}{ Fonte: Os autores (2020) }
\end{tabular}

Com base na divisão das faixas foi possível calcular a matriz de transição, é apresentada na Tabela 2. É possível verificar os percentuais que representam as probabilidades de sair de um estado e ir para um próximo em um passo de tempo.

Tabela 2 - Matriz de transição para o preço do aço para o período analisado

\begin{tabular}{|c|c|c|c|c|c|}
\hline De/Para & $\begin{array}{c}\text { (A) } \\
<-1,67 \%\end{array}$ & $\begin{array}{c}\text { (B) } \\
-1,67 \% \text { a }-0,64 \%\end{array}$ & $\begin{array}{c}(C) \\
-0,64 \% \text { a } 0,38 \%\end{array}$ & $\begin{array}{c}\text { (D) } \\
0,39 \% \text { a } 1,41 \%\end{array}$ & $\begin{array}{c}(\mathrm{E}) \\
>1,41 \%\end{array}$ \\
\hline$(A)<-1,67 \%$ & $50 \%$ & $25 \%$ & $25 \%$ & $0 \%$ & $0 \%$ \\
\hline (B) $-1,67 \%$ a $-0,64 \%$ & $9 \%$ & $32 \%$ & $45 \%$ & $14 \%$ & $0 \%$ \\
\hline (C)-0,64\% a $0,38 \%$ & $0 \%$ & $19 \%$ & $59 \%$ & $19 \%$ & $3 \%$ \\
\hline (D) $0,39 \%$ a $1,41 \%$ & $0 \%$ & $5 \%$ & $74 \%$ & $16 \%$ & $5 \%$ \\
\hline$(E)>1,41 \%$ & $0 \%$ & $0 \%$ & $100 \%$ & $0 \%$ & $0 \%$ \\
\hline
\end{tabular}

Para etapa 4 se faz o uso da matriz de transição onde, os valores são inseridos nos termos das equações lineares apresentados nas equações 7 a 12 são calculadas.

$$
\begin{aligned}
& \pi_{0}=0,5 \pi_{0}+0,09 \pi_{1}+0 \pi_{2}+0 \pi_{3}+0 \pi_{4} \\
& \pi_{1}=0,25 \pi_{0}+0,32 \pi_{1}+0,19 \pi_{2}+0,05 \pi_{3}+0 \pi_{4}
\end{aligned}
$$




$$
\begin{aligned}
& \pi_{2}=0,25 \pi_{0}+0,45 \pi_{1}+0,59 \pi_{2}+0,74 \pi_{3}+1 \pi_{4} \\
& \pi_{3}=0 \pi_{0}+0,14 \pi_{1}+0,19 \pi_{2}+0,16 \pi_{3}+0 \pi_{4} \\
& \pi_{4}=0 \pi_{0}+0 \pi_{1}+0,03 \pi_{2}+0,05 \pi_{3}+0 \pi_{4} \\
& 1=\pi_{0}+\pi_{1}+\pi_{2}+\pi_{3}+\pi_{4}
\end{aligned}
$$

Com a solução do sistema de equações linear e, considerando que uma das primeiras 5 equações é redundante se obtém o resultado apresentado na Tabela 3.

Tabela 3 - Divisão da variação percentual para cada faixa

\begin{tabular}{cccccc}
\hline Faixa & A & B & C & D & E \\
\hline Intervalo & $<-1,67 \%$ & $-1,67 \%$ a & $-0,64 \%$ a & $0,39 \%$ a & $>1,41 \%$ \\
$\begin{array}{c}\text { Prob. Estado } \\
\text { estável }\end{array}$ & $0,64 \%$ & $0,38 \%$ & $1,41 \%$ & \\
\hline & $3,45 \%$ & $18,97 \%$ & $58,62 \%$ & $16,38 \%$ & $2,59 \%$ \\
\hline
\end{tabular}

Com base na solução apresentada, verifica-se que a maior probabilidade, de 58,62\% é de que ocorram variações dentro da faixa $\mathrm{C}$, ou seja, entre $-0,64 \%$ e $0,38 \%$. Da mesma forma, verifica-se que as probabilidades de ocorrência das faixas dos extremos, A e E, são relativamente menores, respectivamente $3,45 \%$ e $2,59 \%$. A partir disso, também foram calculados os tempos de recorrências, apresentados na Tabela 4, onde se verifica que o tempo esperado de retornar ao estado C, uma vez que deixe o mesmo é de 1,7 meses. Já, para o estado E, o tempo de recorrência é 38,7 meses, devido à baixa probabilidade de ocorrência.

Tabela 4 - Divisão da variação percentual para cada faixa

\begin{tabular}{cccccc}
\hline Faixa & A & B & C & D & E \\
\hline $\begin{array}{c}\text { Intervalo } \\
\text { Tempo }\end{array}$ & $<-1,67 \%$ & $-1,67 \%$ a & $-0,64 \%$ a & $0,39 \%$ a & $>1,41 \%$ \\
$\begin{array}{c}\text { esperado de } \\
\text { recorrência }\end{array}$ & 29 & $5,64 \%$ & $0,38 \%$ & $1,41 \%$ & \\
\hline & & & & \\
\end{tabular}

\section{Considerações finais}

No presente artigo se fez aplicação da técnica de Cadeias de Markov para avaliar o comportamento da variação do preço do aço no Brasil. A aplicação se mostrou efetiva, uma 
vez que foi possível calcular as probabilidades de transição entre estados, bem como avaliar as probabilidades de estado estável.

Foi possível determinar as maiores probabilidades de ocorrência dentre as faixas selecionadas, uma vez que o estado estável foi calculado. Se verificou que a faixa intermediária de variação é que mais apresenta probabilidade de ocorrência, 58,62\%. Ao mesmo tempo, também se obteve o tempo esperado de recorrência, sendo de 1,7 mês para esta faixa. As faixas de variação percentual maiores de um mês para o outro se mostraram com probabilidade de ocorrência relativamente mais baixas.

\section{REFERÊNCIAS}

ANDRADE, Eduardo Leopoldino. Introdução à pesquisa operacional: métodos e modelos para análise de decisões. 5. ed. Rio de Janeiro: LTC, 2015.

BOLSON, M.; CECHIN, R.; TOMÉ, F.; BIASUZ R.; CORSO, L. L. Análise do índice de inflação brasileiro utilizando cadeias de markov, 2019

CECHIN, Rafaela Boeira; CORSO, Leandro Luís. High-Order Multivariate Markov Chain Applied In Dow Jones And IBOVESPA Indexes. Pesquisa Operacional, v. 39, n. 1, p. 205-223, 2019.

COLIN, Emerson C. Pesquisa Operacional. Editora: LTC, 2007.

CORSO, Leandro L.; WALLACE, Mark. A hybrid method for transportation with stochastic demand. International journal of logistics research and applications. v. 18, n. 4, p. 342-354, 2015.

CRUZ, Manuel Bravo de Faria. Valores e vectores próprios de cadeias de Markov: Métodos de projecção. 2012. 128 f. Dissertação (Mestrado) - Curso de Engenharia, Faculdade de Engenharia da Universidade do Porto, Porto, 2012. Disponível em: <http://www.rcaap.pt/detail.jsp?id=oai:repositorio-aberto.up.pt:10216/12244>. Acesso em: 10 mar. 2017.

HILLIER, Frederick S.; LIEBERMAN, Gerald J. Introdução à Pesquisa Operacional. 9. ed. Porto Alegre: Amgh, 2013.

RAGSDALE, Cliff T. Modelagem e Análise de Decisão. 6ª edição. São Paulo: Cengage Learning, 2009.

TAHA, Hamdy A. Pesquisa operacional uma visão geral. São Paulo: Pearson Prentice Hall, 2008. 